\title{
Impact of Antimicrobial Stewardship Program (ASP) on Outcomes in Patients with Acute Bacterial Skin and Skin Structure Infections (ABSSSIs) in an Acute- Tertiary Care Hospital
}

Li Wen Loo · Yi Xin Liew · Winnie Lee · Piotr Chlebicki •

Andrea Lay-Hoon Kwa

To view enhanced content go to www.infectiousdiseases-open.com

Received: June 5, 2015 / Published online: September 11, 2015

(c) The Author(s) 2015. This article is published with open access at Springerlink.com

\section{ABSTRACT}

Introduction: Acute bacterial skin and skin structure infections (ABSSSIs) are among the most common infections treated in hospitals, but to date, there has been little information with regards to the implementation of Antimicrobial Stewardship Programs (ASPs) for patients with ABSSSIs. Hence, we aim to evaluate the impact of ASPs on the following outcomes in patients with ABSSSIs: duration of

L. W. Loo and Y. X. Liew contributed equally to this work.

L. W. Loo · Y. X. Liew · W. Lee · A. L. Kwa ( $\square)$

Department of Pharmacy, Singapore General

Hospital, Singapore, Singapore

e-mail: andrea.kwa.1.h@sgh.com.sg

P. Chlebicki

Department of Infectious Diseases, Singapore

General Hospital, Singapore, Singapore

\section{A. L. Kwa}

Emerging Infectious Diseases, Duke-National University of Singapore Graduate Medical School, Singapore, Singapore

\section{A. L. Kwa}

Department of Pharmacy, Faculty of Science,

National University of Singapore, Singapore,

Singapore therapy and hospital stay, 14-day reinfection, infection-related readmissions and mortality.

Methods: A retrospective review of the ASP database was conducted, focusing on selected outcomes (as above) among all patients in whom the institution's ASP recommended a change in antibiotic regimen-de-escalation of the antibiotic based on culture results; discontinuation of the antibiotic; narrowing of the empirical coverage; and intravenous-to-oral (i.v.-to-p.o.) switch between September 2009 and December 2012. Data were expressed as mean \pm standard deviation for continuous variables, and unpaired Student's $t$ test was performed to determine intergroup differences between mean values. For categorical variables, data were presented as number and percentage and analyzed using the $\chi^{2}$ test or Fisher's exact test, as appropriate.

Results: ASP recommended 407 interventions with an overall acceptance rate of $66.8 \%$. ASP interventions significantly reduced median duration of therapy by 2 [from a median (interquartile range, IQR) of $8(6-12)$ days to 6 (4-9) days] and median length of stay by 5 days [from median (IQR) of 12 (5-32) days to 7 (3-18) days]. This led to an estimated total cost 
avoidance of USD 0.7 million. There were no significant differences in the 14-day reinfection, infection-related readmission and mortality rates between patients whose physicians accepted and those who rejected ASP interventions.

Conclusion: Interventions recommended by the ASP in Singapore General Hospital were safe and associated with a significant reduction in duration of therapy and hospital stay. The results of our study have affirmed the role of ASP in optimizing the care of patients with ABSSSI.

Keywords: Acute skin and skin structure infections; Antimicrobial stewardship program; Impact; Outcomes

\section{INTRODUCTION}

Acute bacterial skin and skin structure infections (ABSSSIs) are ubiquitous and among the most common infections treated in hospitals. They differ in severity, and patients who present to the hospital with severe infection or whose infection is progressing despite empirical antibiotics given in outpatient settings should be treated more aggressively [1]. Complicated ABSSSIs represent the more severe end of the spectrum of all ABSSSIs and require timely initiation of appropriate antibiotics. Though many studies have identified the common pathogens involved in ABSSSI, the emergence of strains with resistance to multiple agents has complicated the choice of empirical therapy [2]. Undeniably, antibiotic resistance is mainly driven by overusing antibiotics or by prescribing them inappropriately.

A study conducted in an academic medical center in the USA revealed that despite the gram-positive etiology of most cases of ABSSSIs, two-thirds of their patients were treated with broad-spectrum gram-negative antimicrobial agents and even more received anaerobic therapy [3]. The same study also showed that hospitalizations for ABSSSI are now more common than for community-acquired pneumonia. In a separate matched cohort study, it was found that the management of complicated ABSSSIs in hospitalized patients led to frequent use of potentially unnecessary diagnostic studies, broad-spectrum antibiotic therapy and prolonged treatment courses. This in turn results in a longer length of hospital stay, higher hospitalization charges, increased risk of adverse events from prolonged antimicrobial therapy and higher mortality rates. These findings suggest the need for Antimicrobial Stewardship Programs (ASPs) [4].

ASPs were implemented in various countries in an attempt to control the phenomenon of increasing antimicrobial resistance, especially in developed countries. Studies have shown that ASPs can effectively reduce antibiotic utilization, cost of care and even antimicrobial resistance rates [5]. To date, there has been little information with regards to the implementation and achievements of ASP to patients with ABSSSI. Hence, in this study, we aim to evaluate the impact of ASP on the following outcomes in patients with skin and soft tissue infections: (1) duration of therapy, (2) length of hospital stay, (3) readmission within 30 days of discharge, (4) 14-day reinfection post intervention and (5) mortality within 30 days of ASP audit.

\section{METHODS}

\section{Study Design and Setting}

This is a single-center, retrospective study conducted at Singapore General Hospital (SGH). SGH is Singapore's largest acute-tertiary 
care hospital, with a capacity of 1579 beds. A review of the ASP database was conducted, for interventions made between September 2009 and December 2012. For the purpose of this study, only the following outcomes were selected for analysis: duration of therapy, length of hospital stay, readmissions, 14-day reinfection and mortality. Patients were classified into two groups: (1) those whose primary physicians accepted ASP interventions (accepted group); (2) those whose primary physicians rejected ASP interventions (rejected group). During the study period, the audit was progressively expanded to include the following departments: General Surgery (October 2008), Endocrinology (March 2009), Renal Medicine (April 2009), Colorectal Surgery (October 2009), Plastic Surgery (October 2009), Internal Medicine (March 2010), hospital-wide carbapenem audit (January 2011) and Orthopaedic Surgery (December 2012).

\section{Description of Our ASP [5]}

The ASP team, consisting of an infectious diseases (ID) physician, clinical microbiologist and ID clinical pharmacists, drew up new antibiotic guidelines for surgical prophylaxis as well as empirical treatment of common infections. Evidence for these guidelines was drawn from published guidelines by the Infectious Diseases Society of America (IDSA) and the British Society for Antimicrobial Chemotherapy (BSAC) and was adapted to SGH's microbial susceptibility patterns. These guidelines were approved by the institution's Pharmacy and Therapeutics (P\&T) Committee and endorsed by the Medical Board before they were uploaded on the institution's intranet. The ASP team also drew up an intravenous-to-oral (i.v.-to-p.o.) conversion algorithm (1) to facilitate and encourage the appropriate use of oral formulations and (2) to prevent unnecessary i.v. antibiotic use to reduce costs.

Please refer to Fig. 1 for our ASP workflow. Briefly, all patients who received carbapenems, cefepime, piperacillin/tazobactam or parenteral ciprofloxacin were identified from the pharmacy database on a daily basis and were subjected to the two-stage prospective audit with immediate and concurrent feedback. For the first stage of the review, trained ID clinical pharmacists assessed the appropriateness of antibiotics prescribed and made therapeutic recommendations to optimize drug dosing and also to encourage i.v.-to-p.o. conversions. At the second stage of the review, the complicated cases with diagnostic conundrums were brought up for discussion with an ID physician. The ASP team will follow up on the audited patient cases on the 2nd, 4th (giving a 72-h period for bacterial cultures to be processed) and 7th day (if applicable) of antibiotic prescription, with recommendations for discontinuation, change, dose adjustment, de-escalation of antibiotics or duration of antibiotic use where appropriate. On day 2 of the antibiotic prescription, the ASP team will evaluate the empiric use of audited antibiotics based on the institution antibiotic guidelines as well as antibiogram. As bacterial cultures usually require $48-72 \mathrm{~h}$ to be processed by our microbiology laboratory, the ASP team will do a second review at day 4 , when culture results are reported. At day 4, if the choice of the audited antibiotic is marked as 'appropriate use' by the ASP team, the case will then be followed up with regards to the duration of therapy. As there is no fixed duration of therapy recommended for various ABSSSIs, the ASP team will follow up at days 7, 10 and 14 of therapy to review and assess the wound and to correlate with patients' clinical status before making any intervention. Should the 


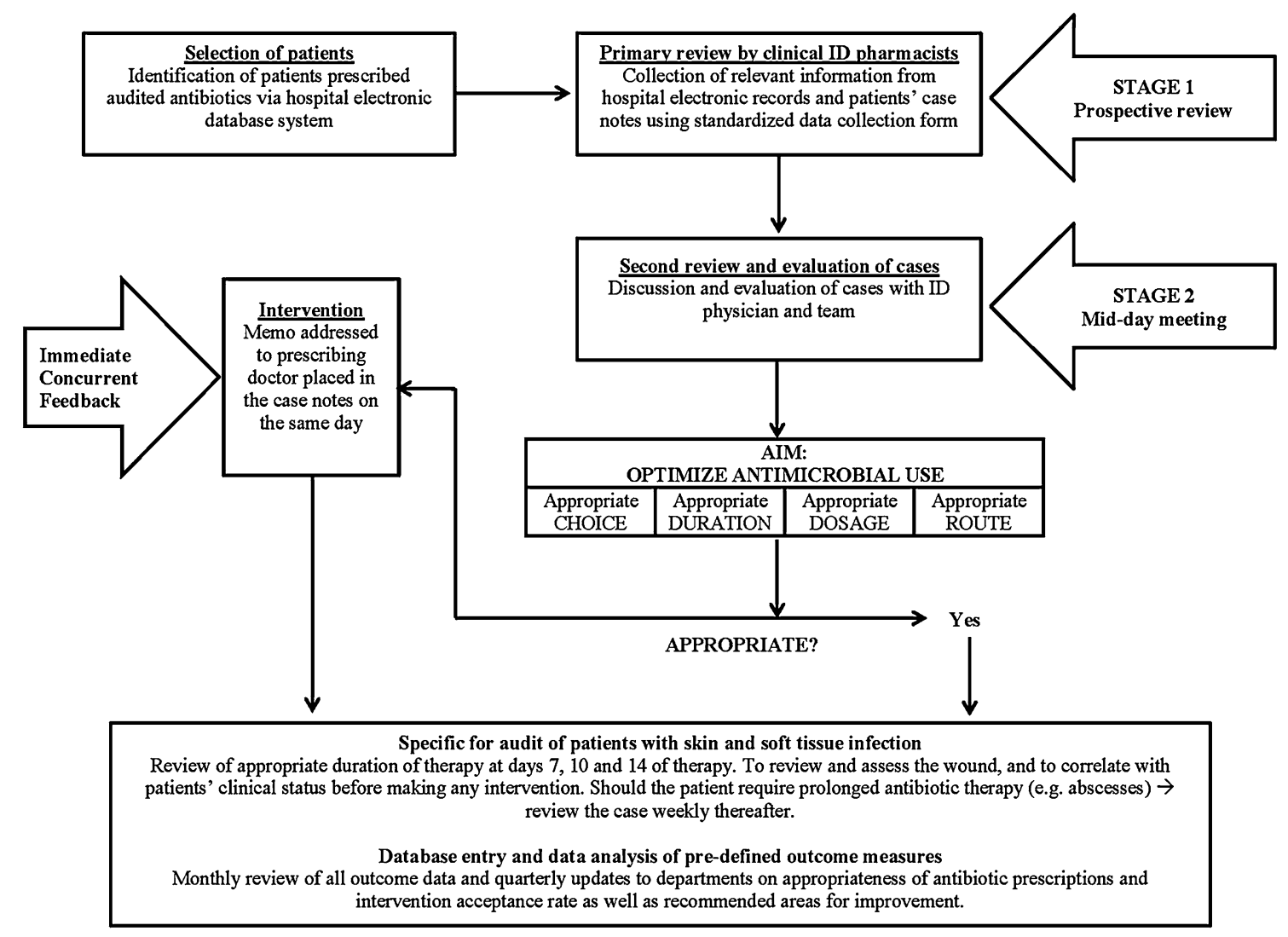

Fig. 1 Schematic diagram of the antimicrobial stewardship program prospective audit with immediate concurrent feedback workflow. ID infectious diseases

patient require prolonged antibiotic therapy (e.g., in patients with abscesses), the ID pharmacists in the ASP team will review the case weekly thereafter. Sometimes, clinical tests (cultures and/or procalcitonin, etc.) were recommended by the ASP team to guide the judicious use of antibiotics. Antibiotics were considered to be inappropriately prescribed if one or more of the following criteria were met: (1) a narrower spectrum antibiotic could be used based on the culture results; (2) there was no infection present (i.e., bacterial colonization or an alternative explanation for the fever present); (3) hospital antibiotic guidelines were not followed without valid reasons; (4) dosage, duration of therapy and/ or empirical treatment choice was suboptimal according to the available guidelines.

\section{Data Collection and Outcomes}

Compliance with or rejection of ASP recommendations was determined via chart review 24 and $48 \mathrm{~h}$ post recommendation as part of the clinical workflow. If the recommendations were adopted after this time frame, they were still considered to be rejected for the purposes of this study. In addition, this study will also aim to estimate the cost avoidance associated with the selected outcomesduration of therapy, length of hospital stay, readmissions, 14-day reinfection and mortality.

\section{Definitions}

For the purpose of this study, only four types of interventions were evaluated as these 
interventions potentially could have a direct impact on the selected outcomes as listed above. These interventions include de-escalation of the antibiotic based on culture results, discontinuation of the antibiotic, narrowing of empirical coverage and i.v.-to-p.o. switch. While the ASP team occasionally recommends additional investigations, infection control measures or ID referral, such interventions in this analysis were not included as they are less likely to have a direct impact on the selected outcomes.

Thirty-day readmissions were defined as admissions within 30 days of the date of discharge. The length of stay (LOS) is defined as the duration of hospital stay starting from the date of the ASP intervention to the date of patient discharge. Mortality was defined as patients who died within 30 days from the date of the ASP audit. Absence or presence of reinfections was evaluated in patients on day 14 post discontinuation of the audited antibiotic in culture-directed therapies. The reinfection must be attributable to the same organism isolated from the same site of infection, with the same susceptibility, which was treated by the preceding course of antibiotic therapy. The classification of ABSSSI severity is adopted from Dryden [2] (Table 1).

\section{Ethics}

The study protocol was approved by the Singhealth Centralized Institutional Review Board (CIRB Ref: 2010/114/E). Informed consent was not obtained from individual patients as the operations of ASP constituted routine clinical practice, and only anonymized data were analyzed.

\section{Statistical Methods}

IBM SPSS Statistics (version 20) was used for all statistical calculations. Data were expressed as mean \pm standard deviation for continuous variables, and unpaired Student's t-test was performed to determine intergroup differences between mean values. For the length of hospital stay post-ASP intervention, data were expressed as median values with range and compared via the Mann-Whitney $U$-test. For categorical variables, data were presented as the number and percentage and were analyzed using $\chi^{2}$ test or Fisher's exact test, as appropriate.

Table 1 Classification of ABSSSI severity [2]

\begin{tabular}{lll}
\hline Category & Clinical features & Management \\
\hline Class 1 & $\begin{array}{l}\text { ABSSSI but no signs or symptoms of systemic toxicity or } \\
\text { comorbidities }\end{array}$ & $\begin{array}{c}\text { Drainage (if required) and oral antibiotics as } \\
\text { outpatient }\end{array}$ \\
Class 2 & $\begin{array}{l}\text { Either systematically unwell or systematically well but } \\
\text { with comorbidity (e.g., diabetes) that may complicate or } \\
\text { delay resolution }\end{array}$ & $\begin{array}{c}\text { Oral or outpatient intravenous antibiotic therapy, } \\
\text { may require short period of observation in } \\
\text { hospital }\end{array}$ \\
& $\begin{array}{l}\text { Toxic and unwell (fever, tachycardia, tachypnea and/or } \\
\text { hypotension) }\end{array}$ & $\begin{array}{c}\text { Likely to require inpatient treatment with } \\
\text { parenteral antibiotics }\end{array}$ \\
& $\begin{array}{l}\text { Sepsis syndrome and life-threatening infection (e.g., } \\
\text { necrotizing fasciitis) }\end{array}$ & $\begin{array}{c}\text { Likely to require admission to ICU, urgent surgical } \\
\text { assessment and treatment with parenteral } \\
\text { antibiotics }\end{array}$
\end{tabular}

ABSSSI acute bacterial skin and skin structure infections, $I C U$ intensive care unit 


\section{RESULTS}

\section{Interventions}

The ASP team recommended a total of 407 interventions (Table 2), with an average acceptance rate of $66.8 \%(272 / 407)$, between September 2009 and December 2012. There were no statistically significant differences in terms of age, gender, previous hospitalization within 3 months and previous antibiotic use within 3 months between the accepted group and rejected group, as defined above (Table 3). The most commonly audited antibiotics were piperacillin/tazobactam (48.4\%), parenteral ciprofloxacin (22.1\%), meropenem (10.8\%) and cefepime $(10.0 \%)$.

\section{Duration of Therapy}

The antibiotic use duration was significantly shorter by 2 days $(P<0.01)$ in the group of patients whose physicians accepted ASP interventions [median (IQR) of 6 (4-9) days] when compared to the group whose physicians rejected ASP interventions [median (IQR) of 8 (6-12) days], as shown in Table 4.

\section{Length of Stay}

The LOS from the ASP intervention and discharge from the hospital was significantly

Table 2 Selected interventions analyzed in this study

\begin{tabular}{lrr}
\hline Intervention & $\boldsymbol{N}$ & $\begin{array}{l}\text { Accepted } \\
{[\boldsymbol{n}(\%)]}\end{array}$ \\
\hline De-escalation based on culture results & 57 & $39(68.4)$ \\
Discontinue antibiotic & 173 & $118(68.2)$ \\
Intravenous-to-oral switch & 118 & $76(64.4)$ \\
Narrowing of empirical coverage & 59 & $39(66.1)$ \\
Total & 407 & $272(66.8)$ \\
\hline
\end{tabular}

shorter by 5 days $(P<0.01)$ in the accepted group [median (IQR) of 7 (3-18) days] than in the rejected group [median (IQR) of 12 (5-32) days]. According to the local Ministry of Health hospital bill size data (updated March 2015), the estimated total cost avoidance from the reduction in length of hospital stay is USD 0.7 million.

\section{Reinfection}

There were 141 patients with positive microbiological cultures. Of these, 4 (8\%) of the 50 patients in the rejected group had reinfection within 14 days, while 2 (2.2\%) of the 91 patients in the accepted group had reinfection within 14 days. However, the difference in the 14-day reinfection rates between the accepted and rejected group was not statistically significant $(P=0.10)$.

One patient in the accepted group had a right foot abscess where pan-sensitive Pseudomonas aeruginosa was isolated intraoperatively. The ASP team had intervened to use oral ciprofloxacin when the wound was improving, and the primary team accepted the intervention. However, in view of the poor glycemic control and slow wound healing, the patient subsequently underwent multiple debridements for source control, and again, intraoperative culture grew pan-sensitive $P$. aeruginosa. The second patient, who had colorectal carcinoma of the sigmoid, complicated with perianal fistula, was admitted for a right buttock abscess (secondary to tumor fistulation). The patient underwent surgical drainage of the abscess and subsequently a second round of wound debridement. The patient was initiated on i.v. cefepime based on intraoperative culture that grew pan-sensitive $P$. aeruginosa. After 10 days of i.v. cefepime, the patient had improved 
Table 3 Patient demographics

\begin{tabular}{llll}
\hline Demographics & Accepted group $(\boldsymbol{N}=\mathbf{2 7 2})$ & Rejected group $(\boldsymbol{N}=\mathbf{1 3 5})$ & $\boldsymbol{P}$ value \\
\hline Mean age (years) & 63.7 & 62.2 & 0.35 \\
Male gender & $152(55.9)$ & $79(58.5)$ & 0.61 \\
Previous hospitalization within 3 months & $140(51.5)$ & $66(48.9)$ & 0.62 \\
Previous antibiotic use within 3 months & $186(68.4)$ & $95(70.4)$ & 0.68 \\
Charlson comorbidity score & & & \\
$1-2$ & 58 & 34 & 0.67 \\
$3-4$ & 66 & 33 & \\
$\geq 5$ & 148 & 68 & \\
Median (IQR) & $5(3-7)$ & $5(2-6)$ & \\
Classification category for ABSSSI severity & & & 0.927 \\
1 & 0 & 52 & \\
2 & 119 & 60 & \\
3 & 119 & 23 & \\
4 & 34 & $3(2,3)$ & \\
Median (IQR) & & & \\
\hline
\end{tabular}

Data are $n(\%)$ unless otherwise stated

$A B S S S I$ acute bacterial skin and skin structure infections, $I Q R$ interquartile range

clinically, and the surgical wound was healing well. The ASP team had intervened to discontinue i.v. cefepime. The intervention was accepted, and patient was subsequently discharged well 4 days thereafter. However, at 5 days post discharge, the patient was readmitted for fever, and her perianal wound was noted to be foul smelling. Wound swab culture grew the same pan-sensitive $P$. aeruginosa, and the patient was re-initiated on another course of i.v. cefepime.

\section{Readmissions}

Of the 383 surviving patients, 24 out of 254 patients $(9.45 \%)$ in the accepted group was readmitted within 30 days from discharge, with a clinical diagnosis of ABSSSI, while 9 out of 129 patients $(6.98 \%)$ in the rejected group was readmitted for ABSSSI. The difference in readmission rates between the two groups was not statistically significant $(P=0.42)$.

\section{Mortality}

Of the 272 patients in the intervention-accepted group, 254 (93.4\%) survived beyond 30 days after discharge, while $129(95.6 \%)$ out of 135 in the intervention-rejected group survived beyond 30 days after discharge. For mortality due to ABSSSI, there were $4(1.47 \%)$ patients in the accepted group and $3(2.22 \%)$ in the rejected group. There was no statistical difference in terms of 30-day all-cause mortality $(P=0.38)$ as well as 30-day mortality due to ABSSSI $(P=0.69)$ between the two groups. 
Table 4 Impact of ASP interventions on the selected outcomes in patients with ABSSSI

\begin{tabular}{llcr}
\hline Outcomes & Accepted group $(\boldsymbol{N}=\mathbf{2 7 2})$ & Rejected group $(\boldsymbol{N}=\mathbf{1 3 5})$ & $\boldsymbol{P}$ value \\
\hline Duration of therapy (days), median (IQR) & $6(4-9)$ & $8(6-12)$ & $<0.01^{*}$ \\
Length of stay (days), median (IQR) & $7(3-18)$ & $12(5-32)$ & $<0.01^{*}$ \\
30-day all-cause mortality & $18(6.60)$ & $6(4.44)$ & 0.38 \\
30-day mortality due to ABSSSI & $4(1.47)$ & $3(2.22)$ & 0.69 \\
\hline & $(\boldsymbol{N}=\mathbf{2 5 4})$ & $(\boldsymbol{N}=\mathbf{1 2 9})$ & 0.42 \\
\hline 30-day readmission due to ABSSSI & $24(9.45)$ & $9(6.98)$ & 0.10 \\
\hline & $(\boldsymbol{N}=\mathbf{9 1})$ & $(\boldsymbol{N}=\mathbf{5 0})$ & \\
\hline 14-day reinfection & $89(97.8)$ & $46(92)$ & $4(8)$ \\
Reinfection absent & $2(2.2)$ & & \\
Reinfection present & &
\end{tabular}

Data are $n$ (\%) unless otherwise stated

$A B S S S I$ acute bacterial skin and skin structure infection, $A S P$ antimicrobial stewardship program, $I Q R$ interquartile range

* Statistically significant $(P<0.05)$

\section{DISCUSSION}

As evident by the results of our study, ASP interventions are important to improve the quality of prescribing and to guide physicians on the appropriate duration of antibiotics without compromising on patient's safety. Moreover, in our institution, ASP reviews commence on the 2nd day of antibiotic prescription, allowing for timely feedback and intervention. Prospective audit as well as feedback intervention is one of the two core ASP strategies recommended by the IDSA [6] that has been shown to be effective.

While guidelines have suggested an average of 5-10-day duration for cellulitis [1] and a longer duration if there are abscesses, more often than not, physicians extend the duration of therapy beyond recommendations in view of poor resolution of the infection. We have shown that our ASP interventions were effective in reducing the median duration of antibiotic therapy by 2 days $(P<0.01)$, as well as reducing the median LOS by 5 days $(P<0.01)$, without compromising the safety of our patients. It was also suggested that limiting the treatment duration seemed to be the most effective method, where antibiotic administration can be modified, to reduce antibiotic resistance as well as the other drug-related deleterious effects such as ADRs [7].

Concomitant with a reduction in the duration of antibiotic therapy, ASP interventions have also effectively reduced the median LOS by 5 days. This is most likely attributed to cases where physicians accepted interventions to discontinue the antibiotic and/ or to switch to oral antibiotics. The prolonged LOS for patients in the rejected group may be explained by the need to complete a prolonged course of parenteral antibiotic therapy, contrary to ASP recommendations. Early discharge from the hospital will result in a reduction in treatment costs and relieve bed crunch problems, but, more importantly, substantially reduce patients' risk of acquiring nosocomial 
infections. Of note, extended duration of antibiotic therapy and a longer duration of hospitalization were associated with a substantially increased risk of Clostridium difficile-associated diarrhea, accounting for $20-30 \%$ of cases of antibiotic-associated diarrhea [8]. C. difficile infection is regarded as an immediate public health threat that requires urgent and aggressive action [9].

One of the more common interventions made by ASP was to encourage physicians to switch to oral therapy, which entails benefits to both the patient and healthcare system. First, it reduces the cost of treatment for the patients as most parenteral antibiotics are more expensive than oral formulations, and there are additional costs associated with preparation and administration of parenteral formulations. More importantly, it reduces healthcare costs by reducing the incidence of catheter-related infections and allowing for shorter hospital stays when patients are discharged with oral antibiotics to complete therapy.

While it may be argued that the patients whose physicians rejected ASP interventions were likely more ill and hence reluctant to switch to an antibiotic of a narrower spectrum or to switch to oral antibiotics, we found no significant differences in the baseline characteristics of patients in the two groups. Of the $33.2 \%$ of cases where ASP interventions were rejected, approximately half of them were rejected because of the physician's personal preference, with no clear reasons indicated. Some possible reasons could be that physicians are not inclined to accept interventions when they deviate from their clinical judgment or they may have the misconception that ASP is driven with the sole purpose to discontinue the use of antibiotics. Contrary to this, ASP is in place to ensure the judicious use of antibiotics and optimize clinical outcomes while minimizing unintended consequences of antimicrobial use rather than merely decreasing antibiotic expenditure [10].

Although not statistically significant, the 14-day reinfection rates were lower in the accepted group, and this could be a result of an appropriate choice, with adequate doses and duration of therapy as recommended by the ASP, thereby leading to better patient outcomes. The 30-day readmission rates due to ABSSSIs were slightly higher in the accepted group $(9.45 \%)$ compared to the rejected group (6.98\%), but this difference was not statistically significant $(P=0.42)$. The majority of these patients had conditions that impair wound healing, such as poorly controlled diabetes mellitus and peripheral vascular diseases, and this in turn explained the recurrent admissions because of ABSSSIs. The 30-day mortality rates for ABSSSIs were comparable between the two groups $(1.47 \%$ in the accepted group, $2.22 \%$ in the rejected group), and this difference was not statistically significant $(P=0.69)$. All seven patients, who died because of ABSSSIs had 'source control' issues where surgical interventions such as amputation were deemed necessary by physicians in a bid to control the infection. Two of these patients had high cardiac risk for surgeries and thus were contraindicated for the needed surgical interventions required to control the infections. The remaining five patients had refused further surgical interventions and opted for conservative management with antibiotics and wound dressings. The ASP team had intervened to discontinue antibiotics in these seven patients as they had received prolonged courses of antibiotics but failed to improve clinically because of the lack of 'source control.' Extending the duration of antibiotics in this group of patients will not benefit them because 
of medical futility. Instead, it puts patients at a higher risk of side effects of the antibiotics, as well as increased risk of $C$. difficile-associated diarrhea, as mentioned above.

Of note, our study revealed that many patients were treated with antibiotics based on wound/skin swab cultures. While swab cultures are easily sampled, they are controversial, especially when obtained from chronic wounds. The culture may be obtained from an uninfected wound and lead to unnecessary antibiotic therapy [11]. The ASP team in our institution had actively intervened to educate the primary team about stopping antibiotics for positive swab cultures from patients with no clinical infective symptoms, and positive swab cultures should not be used to determine whether the wound is acutely infected. Rather, swab cultures may be used to identify potential pathogens in a wound judged to be infected based on clinical criteria in circumstances where tissue biopsy is not possible. Future studies may be conducted to compare patient outcomes where they are initiated on antibiotic therapies based on tissue biopsy compared to those who are treated based on swab cultures.

With a relatively large sample size and involvement of various specialties, the results of our study can be applied to a variety of healthcare settings worldwide. However, the retrospective nature of our study limits the amount of information we have with regards to differences in the severity of diseases and/or presence of comorbidities that could potentially affect outcomes. Nevertheless, with similar baseline demographics between the two groups, the possibility of such differences affecting the outcomes is low.

\section{CONCLUSIONS}

Interventions recommended by the ASP in SGH were safe and were associated with a significant reduction in the duration of antibiotic therapy as well as a reduction in the length of hospital stay. Although ABSSSIs are prevalent, little has been done to improve the quality of prescribing antibiotics for its management. The results of our study have affirmed the role of ASP in optimizing the care of patients with ABSSSIs.

\section{ACKNOWLEDGMENTS}

This supplement was not sponsored by outside commercial interests. No source of funding was received for this study or publication of this article. All named authors meet the International Committee of Medical Journal Editors (ICMJE) criteria for authorship for this manuscript, take responsibility for the integrity of the work as a whole and have given final approval for the version to be published.

Conflict of interest. Li Wen Loo, Yi Xin Liew, Winnie Lee, Piotr Chlebicki and Andrea Lay-Hoon Kwa have nothing to disclose.

Compliance with ethics guidelines. The study protocol was approved by the Singhealth Centralized Institutional Review Board (CIRB Ref: 2010/114/E). Informed consent was not obtained from individual patients as the operations of the ASP constituted routine 
clinical practice, and only anonymized data were analyzed.

Open Access. This article is distributed under the terms of the Creative Commons Attribution-NonCommercial 4.0 International License (http://creativecommons.org/licenses/ by-nc/4.0/), which permits any noncommercial use, distribution, and reproduction in any medium, provided you give appropriate credit to the original author(s) and the source, provide a link to the Creative Commons license, and indicate if changes were made.

\section{REFERENCES}

1. Stevens DL, Bisno AL, Chambers HF, et al. Practice guidelines for the diagnosis and management of skin and soft-tissue infections. Clin Infect Dis. 2005;41:1373-406.

2. Dryden MS. Complicated skin and soft tissue infection. J Antimicrob Chemother 2010; 65 Suppl 3: iii35-iii44.

3. Jenkins TC, Sabel AL, Sarcone EE, Price CS, Mehler PS, Burman WJ. Skin and soft tissue infections requiring hospitalization at an Academic Medical Center: opportunities for antimicrobial stewardship. Clin Infect Dis. 2010;51(8):895-903.

4. Hatoum HT, Akhras KS, Lin SJ. The attributable clinical and economic burden of skin and soft tissue structure infections in hospitalized patients: a matched cohort study. Diagn Microbiol Infect Dis. 2009;64:305-10.

5. Liew YX, Lee $\mathrm{W}$, Loh JCZ, et al. Impact of an antimicrobial stewardship programme on patient safety in Singapore General Hospital. Int J Antimicrob Agents. 2012;40:55-60.

6. Dellit TH, Owens RC, McGowan JE, et al. Infectious Diseases Society of America and the Society for Healthcare Epidemiology of America guidelines for developing an institutional program to enhance antimicrobial stewardship. Clin Infect Dis. 2007;44:159-77.

7. Hayashi Y, Paterson DL. Strategies for reduction in duration of antibiotic use in hospitalized patients. Clin Infect Dis. 2011;52(10):1232-40.

8. Cohen SH, Gerding DN, Johnson S, et al. Clinical practice guidelines for Clostridium difficile infection in adults: 2010 update by the Society for Healthcare Epidemiology of America (SHEA) and the Infectious Diseases Society of America (IDSA). Infect Control Hosp Epidemiol. 2010;31(5):431-55.

9. Antimicrobial Resistance Threats in the United States. U.S. Department of Health and Human Sciences, Centers for Disease Control and Prevention. 2013.

10. Mertz D, Koller M, Haller P, et al. Outcomes of early switching from intravenous to oral antibiotics on medical wards. J Antimicrob Chemother. 2009;64:188-99.

11. Drinka P, Bonham P, Crnich CJ. Swab culture of purulent skin infection to detect infection or colonization with antibiotic-resistant bacteria. JAMDA. 2012;13:75-9. 